\title{
Técnicas de desarrollo organizacional para la actualización tecnológica del docente en instituciones de educación superior
}

\author{
Carlos Pacheco-Ruíz' ${ }^{1}$, Claudia Rojas-Martínez', William Niebles-Nuñez ${ }^{1}$ y Hugo G. Hernández-Palma² \\ (1) Universidad de Sucre, Programa de Administración de Empresas, Sincelejo-Colombia. \\ (correo-e: carlos.pacheco@unisucre.edu.co; claudia.rojas@unisucre.edu.co; william.niebles@unisucre.edu.co) \\ (2) Universidad del Atlántico, Programa Administración de Empresas, Barranquilla-Colombia \\ (correo-e: hugohernandezpalma@gmail.com)
}

Recibido Feb. 18, 2020; Aceptado Abr. 20, 2020; Versión final Jun. 3, 2020; Publicado Oct. 2020

\begin{abstract}
Resumen
Esta investigación caracteriza las técnicas de desarrollo organizacional para la actualización tecnológica del docente en instituciones públicas de educación superior de la región caribe (Colombia). La metodología fue descriptiva, con un diseño no experimental de campo, y la población estuvo conformada por cuarenta docentes. La técnica de recolección de datos fue un cuestionario, integrado por doce ítems, con diferentes alternativas tipo escala Likert. El cálculo de la confiabilidad fue de 0.80 con el método de Cronbach, comprobando que el instrumento es confiable. Los datos fueron tabulados e interpretados estadísticamente mediante frecuencia absolutas y relativas, agrupados por dimensión e indicador. Los resultados muestran que las instituciones aplican técnicas de desarrollo organizacional en sustento del aprendizaje organizacional, gestión del conocimiento, equipos de trabajo y socialización. En conclusión, en las universidades públicas de la región caribe se promueven dichos procesos a partir de la integración del docente con los proyectos educativos, permitiendo garantizar la formación continua del docente.
\end{abstract}

\section{Organizational development techniques for the technological updating of the faculty in higher education institutions}

\begin{abstract}
This research study characterizes organizational development techniques for technology updating of professors teaching at public higher education institutions from the Caribbean region in Colombia. The methodology was descriptive with a non-experimental field design. The population consisted of forty professors. The data was collected using a questionnaire, composed of twelve items, having different Likert scale alternatives. The reliability was 0.80 (Cronbach method), proving that the instrument was reliable. Data were tabulated and interpreted statistically by absolute and relative frequencies, grouped by dimension and indicator. The results showed that institutions apply organizational development techniques to support organizational learning, knowledge management, teamwork, and socialization. In conclusion, it can be said that these processes are promoted by integrating professors with educational projects, which ensure the continuous professional training of professors at public universities in the Caribbean region.
\end{abstract}




\section{INTRODUCCIÓN}

En la actualidad existen organizaciones realizando cambios mediante el análisis de su estructura, lo cual incluye la adaptación a procesos inherentes a sus actividades. Algunas tratan los cambios como algo que ocurre de manera accidental involucrando las actividades de cambio generadas como proactivas y con determinados propósitos; sin embargo, al surgir la tendencia del desarrollo organizacional es importante asumirlo como una actividad intencional orientada hacia la meta. Destacando que el mundo actual se caracteriza por un cambio constante en el ambiente, el mismo envuelve a las organizaciones con su proceso dinámico, exigiendo cierta capacidad de adaptación como condición básica de supervivencia.

Consideran Viloria et al (2020), que siendo el hombre un ser productivo vinculado al ambiente institucional y además orientado al cumplimiento de objetivos, requiere contar con una estrategia para obtener los procesos esperados; ajustándose el trabajo, a los objetivos, propósitos y metas establecidas en la organización, en el marco de su visión y misión. Bajo este contexto, el éxito o fracaso de una organización se sustenta por situaciones donde el personal realiza o deja de realizar actividades, este es el caso de las instituciones de educación superior, que han estructurado un proceso de cambio planeado, a lo cual Hernández, Parra, et al (2018), sostienen que estos procesos se ejecutan para orientar el comportamiento de los individuos y grupos en función de las líneas de acción que deben ir ejecutando para converger en el ambiente institucional. Esto incide en que los cambios tecnológicos, sociales y económicos existentes en la sociedad, hayan creado la necesidad de generar planes de desarrollo que le permitan competir con el mercado global, por lo cual deben contar con un personal capacitado para adaptarse a los nuevos procesos asumidos por las instituciones y de esta manera cumplir con éxito los objetivos establecidos por sus líderes.

Guizar (2013), al igual que lo plantean García, Durán, Parra, y Martínez (2018), sostienen que un hecho cierto de las organizaciones es que en estas se asume el desarrollo en relación con las transformaciones que emergen en el ambiente externo y que de diferentes maneras afecta e influye en las modificaciones internas, que deben irse vinculando para dar respuesta a las necesidades. En este orden de ideas, se evidencia que las instituciones de educación superior a través de sus programas de desarrollo organizacional vinculen acciones y actividades hacia la gestión del conocimiento en tecnologías, actualizaciones tecnológicas, capacitación técnica, manejo de sistemas operativos entre otros. Lo cual debe responder a los planes estratégicos, diferentes orientaciones en el mercado educativo, dado que es necesario que los estudiantes cuenten con las alternativas de comunicación a través de los sistemas digitales, que le provea el docente.

Atendiendo a las consideraciones anteriores, se vincula la actualización tecnológica, como lo proponen Bates y Sangrá (2011), haciéndose necesario que los trabajadores busquen la adaptación al proceso, aplicando herramientas estratégicas, y de esta manera continuar formando parte de esta aldea global, es así como las tecnologías de información, en opinión de Hernández, et al (2019), se determinan como método orientado a procesar, transmitir, comunicar y evaluar información valiosa desde cualquier punto geográfico, de forma oportuna, además de pertinente a una materia en específico.

Indudablemente que, en la gestión de los programas de desarrollo organizacional, las instituciones de educación superior deben vincular procesos de capacitación al docente, gestionando el conocimiento hacia unas tendencias globales, pasando de las acciones presenciales hacia el apoyo virtual, respecto a las plataformas virtuales, uso de webinar, aplicaciones Web, entre otras. Destacando que hoy en día en pleno siglo XXI; la tecnología en la era de la información y la comunidad crea un entorno social con un amplio proceso comunicativo, el cual se construye de forma implícita como un espacio de aprendizaje, que promueve la autonomía de los individuos, capaz de incrementar las fuentes de información para desarrollar ciertos tipos de conocimiento y técnicas, según lo enunciado por Moreno et al (2018), por lo cual las instituciones de educación superior públicas deben establecer líneas de acción que los lleven hacia ese campo.

Asimismo, los docentes universitarios no pueden desfasarse con respecto al entorno tecnológico moderno, por lo que su obligación es la de actualizarse y capacitarse en todas las áreas correspondientes, con el fin de ofrecer un ambiente de educación a sus alumnos con una visión de la realidad actual, pues los estudiantes de ahora son los profesionales del futuro, así Rivera et al (2019), enuncian que los educadores deben estar en contacto con todas las herramientas que puedan potenciar sus habilidades, por lo cual las IES, deben contextualizar los procesos para gestionar el conocimiento, y establecer acciones tecnológicas para que el docente tenga el control de las aplicaciones para manejar las comunidades de aprendizaje, los objetos virtuales de aprendizaje (OVA), el cual se enlaza en los programas de desarrollo organizacional, lo que vincula el manejo oportuno de la información, como elemento esencial para el éxito de las sociedades actuales.

Todo esto ha generado cambios a lo largo del tiempo en la cultura universitaria, fomentando el acceso y desenvolvimiento de los estudiantes de la mano de la tecnología, provocando una necesidad de formación actualizada y continua, con calidad y flexibilidad; asimismo promueve una transformación de la función 
docente, y la gestión universitaria descentralizada. Por esto, se suscitan las investigaciones multidisciplinarias con carácter grupal, aunado a la presión competitiva, en Kaware, y Sain, (2015); dichas investigaciones abren nuevas vías de aprendizaje, modificando conceptualmente el rol del profesor, tomando en cuenta que la posibilidad de acceder a información actualizada hace que el docente focalice sus esfuerzos en el aprendizaje, buscando promover procesos de aprendizaje con la finalidad de orientar al estudiante hacia la creación de su propio conocimiento a partir del conjunto de recursos de información disponibles.

En este orden ideas, Matukhin y Zhitkova (2015), discuten sobre tecno formación, y en su concepción se implica la capacidad de utilizar las herramientas tecnológicas como recursos que se complementen con los recursos educativos convencionales; esto es algo complejo que ofrece una diversidad de aprendizaje, el desarrollo de las habilidades para usar la herramienta es una primera parte, puesto que para manejar los programas o las maquinas, requieren un aprendizaje especifico de una habilidad. Por ende, el aspecto complejo es el poder generar conocimiento a partir del uso de los recursos tecnológicos, propiciando la necesidad de que los educadores tengan la capacidad de plantear modelos de aprendizaje empleando las TIC como canal de información, a la vez como un constructor de estructuras educativas para desarrollar este nuevo conocimiento; también se crea la necesidad de espacios acordes para la aplicación de estos procesos.

Dentro de estas perspectivas, se involucran las universidades colombianas, las cuales están enlazadas en un procesos de transformación continua en pro de implementar herramientas tecnológicas acordes a las necesidades de los integrantes de la comunidad universitaria, generándoles beneficios, entre ellos: la inscripción electrónica, construcción del horario de clases, consulta de horarios académicos, publicación de investigaciones, y el uso de redes sociales como medio de comunicación y publicitar, u ofertar cursos virtuales que complementen un modelo de educación presencial, semi-presencial o a distancia. Esto con la finalidad de abarcar más estudiantes en territorios lejanos, así como las opciones de disponer de recursos educativos como base de datos y portales para complementar sus bibliotecas dando espacio a que los usuarios puedan gestionar su tiempo, haciendo uso del recurso desde diferentes espacios al alcance de un clip.

Esto sugiere promover en los estudiantes capacidades para fomentar la gestión estratégica tecnológica y organizacional, propiciando su interrelación con el contexto y los stakeholders. Indudablemente, la idea principal es que dichos programas cumplan con parámetros de calidad y respondan a las necesidades de Colombia para ser competitivo en este mundo globalizado. En este orden de ideas las universidades colombianas se encuentran con la oportunidad de ser más selectas y además de buscar un mayor posicionamiento como institución a niveles regionales y nacionales; estas se han visto en la necesidad de estar al nivel de los más recientes desarrollos tecnológicos en función de ofrecer a sus usuarios, que en este caso son los estudiantes, las herramientas necesarias para alcanzar objetivos profesionales que atiendan a sus necesidades en el mundo de hoy, lo que implica involucrarse en procesos tecnológicos de vanguardia.

En este sentido, contextualizar desde los programas de desarrollo organizacional, la actualización tecnológica pone de manifiesto la importancia que tiene la tecnología como un recurso de doble vía, es decir, es un medio para el desarrollo, pero además un espacio de aprendizaje al igual que el hecho de estar en contacto y desarrollar sus competencias tecnológicas las cuales, se presentan como herramientas ante el logro de sus objetivos profesionales. A partir de esto, las universidades concebidas como organizaciones que buscan mantener su posicionamiento inquieren adaptar las herramientas tecnológicas en función de que estas sean coadyuvantes para volverse organizaciones vanguardistas, visualizando como los avances tecnológicos han ido ocupando sus espacios en el quehacer académico.

El contenido programático en las universidades se encuentra inmerso el componente de educación virtual, a distancia, el cual ha tenido un auge en la forma de capacitarse, en pro de la gestión del tiempo de cada participante, por lo tanto, las universidades han tenido que incluir esta metodología para ser competitivo, promoviendo el acompañamiento del estudiante en el caso de los programas, presenciales con apoyo del aula virtual, programas semi-presenciales, o a distancia completamente. De este modo, Moreno et al (2018), consideran que el rol del docente se ha ido transformado en las últimas décadas, paralelamente a la utilización de las herramientas tecnológicas, vistas hoy en día como una necesidad, pues manejar los sistemas de información que permitan construir un espacio de educación virtual deben ser empleados de forma eficiente por los profesores en su dimensión docente y en su dimensión investigativa. Por ende, consideran Villarreal García, Hernández y Steffens (2019), la realidad, que desde las universidades deben diseñarse y gestionarse programas de capacitación para desarrollar las competencias tecnológicas en los docentes quienes construyen desde las aulas virtuales los cursos en línea, en este sentido, necesitan apoyo, orientación.

Asimismo, Moreno et al (2017), consideran que lo más relevante es la formación en el manejo de la más actualizada tecnología (que se encuentre al alcance de la institución), como medio o herramienta de educación y de aprendizaje, de manera que sea un proceso bidireccional tanto para el uso de las herramientas 
tecnológicas en el estudiante, como por parte del docente involucrar estas herramientas para desarrollar estrategias de aprendizaje dentro de la dinámica educativa.

En virtud de lo anteriormente expuesto, las universidades públicas colombianas deben crear y proyectar el desarrollo del conocimiento, promover la actualización tecnológica, alinear a los docentes hacia las nuevas plataformas virtuales, al manejo de los OVA, de esa manera gestionar en forma coherente los objetivos institucionales asociados a las tendencias del nuevo milenio, por otra parte, también la formación del docente debe asegurar la continuidad de los procesos sociales inmersos en los constantes cambios, involucrando además; un potencial de transformación de la sociedad, no sólo por el conocimiento capaz de generar, sino por interactuar con el entorno para construir agrupaciones de conocimientos orientándose a la búsqueda de la excelencia organizacional y poder desenvolverse de manera efectiva. Ello permite contribuir al desarrollo económico incrementando la productividad de las instituciones, dirigiendo y promocionando la innovación, estimulando la formación continua, la cual emerge apoyada en técnicas de desarrollo organizacional.

\section{OTROS ANTECEDENTES}

Hay una serie de antecedentes adicionales los que se hace necesario detallar dado que sustentan la temática y son indispensables para documentar con mayor profundidad las técnicas de desarrollo organizacional las cuales son: i) técnicas de desarrollo organizacional; ii) aprendizaje organizacional; iii) gestión del conocimiento; iv) construcción de equipos; y v) socialización.

\section{Técnicas de desarrollo organizacional}

Los agentes de cambio que desean construir una institución, están orientados hacia el enfoque de desarrollo organizacional, quienes se centran en fundar una organización basada en un conjunto profundamente sostenido de valores y una cultura fuerte, tomando en cuenta la percepción de los líderes organizacionales, quienes atraen a otros que comparten su pasión sobre la visión y estrategias, para lograr que sus organizaciones destaquen en sus industrias, a su vez contribuyan con sus comunidades, además de ser rentables. A continuación, se muestran en la figura 1 las condiciones necesarias para aprovechar las técnicas de desarrollo organizacional
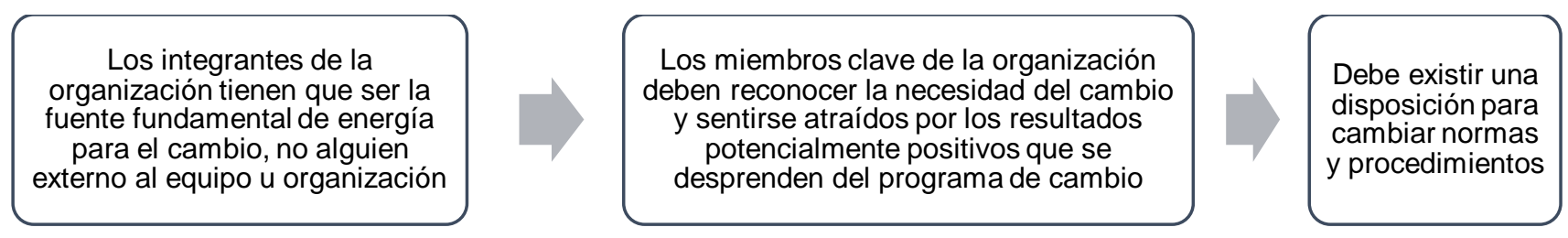

Fig. 1. Condiciones para las técnicas de desarrollo organizacional. Adaptado de Guizar (2013)

De acuerdo con esto, para iniciar un cambio es necesario poseer una visión del estado futuro de la organización, puesto que las resistencias a los cambios y la inercia de la organización se deben a que esta se encuentra atrapada en sus propios paradigmas y rutinas, por lo cual se hace necesario "descongelar" el paradigma, según expresa Schein (1985); Citado por Guizar (2013) este descongelamiento debe ser un requisito de cualquier proyecto de cambio. Asimismo, Alles y Rodríguez (2011), opinan que la visión está precisamente destinada a comprometer la participación y el espíritu de cada uno de los miembros de la organización. La visión se puede concebir como lo que puede hacer una organización, utilizando una ruta para el rumbo futuro y generar emoción sobre ese camino futuro.

Viloria et al (2019), han manifestado que es necesario introducir estas técnicas en las instituciones de educación superior para identificar las necesidades, sus oportunidades, asociadas al camino por recorrer para poder llegar a cubrir las expectativas de sus integrantes, de acuerdo a ello se determinaron las acciones a seguir, con la intención de promover la innovación tecnológica en materia de TIC, favoreciendo la creación de nuevos entornos comunicativos y expresivos, ellos abren la posibilidad de desarrollar nuevas experiencias formativas, expresivas, educativas, posibilitando la realización de diferentes actividades: aprendizaje organizacional, gestión del conocimiento, construcción de equipos y socialización.

\section{Aprendizaje organizacional}

En este orden de ideas, Fiol y Lyles (1985), citado por Cummings y Worley (2014) definen el aprendizaje organizacional como un proceso para mejorar las acciones organizacionales a través de la adquisición y desarrollo de nuevos conocimientos, además de las capacidades, asociadas a las necesidades de las instituciones, en este caso las de educación superior colombianas, las mismas orientadas a generar procesos continuos que les permita mantener en el mercado educativo. 
Para Robbins y Judge (2013), una organización de aprendizaje es aquella que ha adquirido la capacidad continua de adaptarse y cambiar; así como aprenden los individuos, de la misma manera lo hacen las organizaciones. Por lo tanto, las organizaciones de aprendizaje utilizan el aprendizaje de doble ciclo. Cuando se encuentra un error, se corrige de manera tal que implica la modificación de los objetivos, políticas, procesos y rutinas estándares de la organización. En relación con esto último, Viloria et al (2020), asumen que las organizaciones de aprendizaje utilizan conceptos previos de Comportamiento Organizacional como, cultura organizacional, la organización sin fronteras, el conflicto funcional y el liderazgo transformacional. Aunado a ello la inteligencia organizacional no es la suma de las inteligencias de los individuos que forman la organización, es la capacidad de una organización de crear conocimiento y de usarlo para adaptarse estratégicamente a su mercado, es decir los estudiantes.

\section{Gestión del conocimiento}

Cuando una serie de personas empiezan a trabajar en grupo, al principio se suelen producir problemas de coordinación, cuando pasa un tiempo, se van afinando los procesos y cada vez se realiza mejor la tarea. Esto es aprendizaje organizativo, aprender juntos a resolver problemas con una efectividad determinada, el aprendizaje organizativo es la base de una buena Gerencia del Conocimiento, esta es la base para la generación de Capital Intelectual y las capacidades organizativas. Para Nonaka y Takeuchi (2007), la gestión del conocimiento es una disciplina emergente que tiene como objetivo generar, compartir y utilizar el conocimiento tácito (Know-how) y explícito (formal) existente en un determinado espacio, para dar respuestas a las necesidades de los individuos y de las comunidades en su desarrollo.

Según Sánchez García, Steffens y Hernández (2019), el aprendizaje organizacional se percibe como mecanismos clave para el fortalecimiento de una región o espacio en relación con las visiones de futuro que van a determinar sus planes estratégicos de desarrollo a mediano y largo plazo. Además, en las IES colombianas, el conocimiento necesita de un contexto compartido, un lenguaje compartido cuya función consiste en ser un medio para crear significados, que permiten a las personas entender y comprender el contexto de los otros, de sí mismos actuando de acuerdo con dicho conocimiento. Por otro lado, producto de la misma interacción se producen cambios, creando nuevos contextos o espacios de conocimiento y aprendizaje, orientado a la promoción del proceso educativo a través de la investigación.

\section{Construcción de equipos}

Uno de los aspectos fundamentales para el desarrollo organizacional, es el potencial humano, en este sentido las organizaciones cada vez confían más en equipos para desarrollar tareas de trabajo. La construcción de equipos utiliza actividades de grupo de mucha interacción para incrementar la confianza y la franqueza entre los miembros del equipo. De esta manera, Robbins y Judge (2013) considera que la construcción de equipos se puede aplicar en el interior de grupos o a nivel de relaciones entre grupos cuando las actividades son interdependientes. Como resultado, el interés se relaciona con las aplicaciones a las familias organizacionales (grupos de mando), así como a comités, equipos de proyectos, equipos autoadministrados y grupos de tareas. Asimismo, las actividades consideradas en la construcción de equipos suelen incluir la fijación de metas, el desarrollo de relaciones interpersonales entre los miembros del equipo, el análisis de funciones para definir el papel y responsabilidades de cada miembro, además el análisis del proceso de equipo. Dicha construcción puede insistir en ciertas actividades o excluirías, de acuerdo con el propósito del esfuerzo del desarrollo y los problemas específicos que enfrenta el equipo, para Robbins y Judge (2013), la construcción de equipos trata de utilizar una gran interacción entre los miembros para incrementar la confianza y la franqueza.

Otra forma de definir la construcción de equipos según lo planteado por Hernández, Jiménez et al (2018), y Martínez, Steffens, Ojeda y Hernández (2018), es precisarla como las actividades creadas para hacer que el equipo ascienda en la curva de desempeño, la meta de muchos métodos de construcción de equipos es cambiar la cultura de la organización. Por esto, en las universidades de la región caribe se está fomentando la formación de equipos de trabajo como base para el desarrollo organizacional, dado que este facilita la tecno formación orientada al rendimiento institucional, a su vez se traduce a un mayor desempeño del docente.

\section{Socialización}

La socialización es un proceso mediante el cual una persona conoce los valores, normas y conductas requeridas que le permiten participar en la organización como miembro de esta. Independientemente de lo bueno que sea el trabajo de reclutamiento y selección que ponga en marcha la organización, los nuevos empleados no están plenamente adoctrinados en la cultura de la organización. Por tanto, tal vez sea de mayor importancia que, al no estar familiarizados con la cultura organizacional, los nuevos empleados podrían perturbar las creencias y costumbres que se han desarrollado. Por tanto, la organización deseará ayudar a los nuevos empleados a adaptarse a su cultura. Este proceso de adaptación recibe el nombre de adaptación. 
Asimismo, considera Durán (2014) que la rapidez y eficacia de la socialización determinan la lealtad, el compromiso, la productividad de los empleados, así como su permanencia o salida. De tal manera que la estabilidad, sumada a la eficacia de las organizaciones dependerán de la habilidad que tengan éstas para socializar a sus componentes.

Así como lo manifiestan Parra, Visbal, Durán, y Badde (2019), la transmisión de la cultura organizacional de los administradores y empleados superiores a los nuevos empleados, en busca de proporcionarles el conocimiento y las habilidades sociales necesarias para desarrollar las funciones dentro de la organización, además del desarrollo de las tareas organizacionales con éxito, lo cual permita fomentar el crecimiento organizacional. Este último, es un aspecto determinante para el desarrollo de una organización en todos sus niveles, por lo que al respecto Durán (2014), establece que la socialización brinda los medios por los cuales las personas aprenden los aspectos internos de una organización desde el momento de unirse a la misma.

Dentro del marco anterior se incluye el aprender los valores empresariales, además de las reglas, procedimientos y normas organizacionales dentro del grupo de trabajo, los departamentos, organigrama empresarial y fomentar las relaciones sociales de trabajo. Asimismo, Duran expresa que, al desarrollar las competencias necesarias para realizar los deberes de un puesto, convierte este proceso en bidireccional, debido a que el sujeto recibe influencias del medio, mientras influye sobre este y sus participantes como, por ejemplo, compañeros del mismo departamento, miembros que conforman el equipo, personal superior, etc. Por esto, en las universidades públicas de la región caribe se requiere promover procesos de socialización a partir de la integración del docente con los proyectos educativos, lo que fomenta y permite a la institución garantizar la formación continua de su personal docente, lo cual será por consiguiente retransmitido a los estudiantes. Considerando así, como agentes de socialización a las instituciones de educación superior y a aquellos individuos que tienen las atribuciones necesarias para valorar el cumplimiento de las exigencias de la sociedad, y que a su vez disponen del poder suficiente para imponerlas.

\section{METODOLOGÍA}

El estudio se califica como una investigación de tipo descriptiva, porque pretendió caracterizar las técnicas de desarrollo organizacional para la actualización tecnológica del docente en instituciones de educación superior mediante la revisión y determinación de dichas técnicas. En cuanto al diseño de la investigación, en este estudio se utilizó un diseño no experimental, transversal, esto debido a que las variables no fueron manipuladas por los investigadores, y la recolección de datos se realizó en un único momento, obteniendo de esta forma una sola medición. En cuanto a la población, la misma estuvo constituida por cuarenta (40) docentes de distintas universidades públicas de la región caribe colombiana; para seleccionar dicha población, los investigadores establecieron características asociadas con la delimitación de la investigación, tales como; que la población está constituida por docentes de planta (horario a tiempo completo), que tuviesen más de 5 años en la institución y formar parte de las facultades de ciencias económicas, administrativas y contables de las instituciones de educación superior en estudio. Por lo tanto, siguiendo estas características, se consideraron 15 personas de la universidad de Sucre y 25 de la universidad del atlántico. A este respecto, el personal incluido dentro del contexto del universo a estudiar presenta un carácter finito, determinado y accesible de manejar, por esa razón la muestra fue igual a la población, por lo cual se utilizó un censo poblacional, el cual indica que se toma a todos los integrantes del contexto.

Para ejecutar el estudio se utilizó un cuestionario estructurado con alternativas de respuesta tipo likert. En el cual se definieron un conjunto de preguntas, diseñadas sobre los aspectos que conciernen en la investigación, para su contestación por la población. Asimismo, para la aplicación de la técnica se diseñó un cuestionario orientado a determinar las técnicas de desarrollo organizacional utilizadas en las IES pública de la costa caribe colombiana, para promover la actualización tecnológica en los docentes, está integrada por 12 afirmaciones: 1) la institución se orienta a la búsqueda de mayor eficiencia organizacional; 2) el aprendizaje organizacional en la institución proporciona oportunidades para alcanzar soluciones efectivas; 3) la institución tiene la capacidad para adaptarse a cambios; 4) recibe respuesta de la institución a sus necesidades docentes de desarrollo del conocimiento; 5 ) la institución dispone al docente en forma clara y eficaz los conocimientos explicativos de los procesos que debe ejecutar; 6 ) la institución gestiona la actualización tecnológica a través del conocimiento y promueve el mejor funcionamiento de esta; 7) la institución promueve actividades grupales para incrementar la confianza en el equipo; 8) las actividades para la construcción de equipos incluyen la fijación de metas; 9) las actividades para formación docente en actualización tecnológica, refuerzan los niveles de conocimiento grupal; 10) la institución provee de inducción para conocer los valores y normas requeridas para ser miembro de esta; 11) no familiarizarse con la cultura organizacional, podría perturbar las creencias y costumbres que se han desarrollado; 12) la institución promueve la adaptación de sus miembros a la cultura de esta. Para formular los ítems se hizo un análisis del objetivo, se subdividió en indicadores, y por cada uno, se establecieron tres ítems, en este sentido cada uno de los sujetos debería seleccionar la opción pertinente, la escala de opciones viene dada por las alternativas: siempre, casi siempre, a veces, casi nunca y nunca. 
Después de construir el instrumento se aplicó la validez de contenido, que se sustenta en la necesidad de discernimiento y juicios independientes entre expertos, se seleccionaron siete especialistas en el área de gestión y desarrollo organizacional, gerencia educativa y metodología, con el fin de obtener opinión sobre el contenido del instrumento, en el mismo se hicieron observaciones respecto a la redacción y coherencia de 4 ítems. Pero el juicio determinó que el instrumento era válido. En otro sentido se debía determinar la confiabilidad de este, por lo cual se procedió a aplicar una prueba piloto, seleccionando a un grupo de 10 docentes con características similares a la población, se hizo la encuesta se procedió a la tabulación con el programa SPSS, y se calculó la confiabilidad, con el procedimiento de Alpha Cronbach. Este se aplica para los cuestionarios con escala tipo Likert. Dando como resultado rtt. 0.80 . El valor establece un intervalo que oscilan entre 0 y 1 entendiéndose el coeficiente 0 como nula confiabilidad y 1 como confiabilidad total, determinando alta confiabilidad.

En virtud de que la investigación es de tipo descriptiva, de campo, en esa línea los resultados obedecen al logro de una perspectiva global de toda la suma de una serie de datos agrupados dando estos como resultado la construcción de la tabla, que posibiliten visualizar los datos iniciales, transformando estos en términos porcentuales en cada ítems, indicador y respectiva dimensión; obteniéndose de esta manera estimaciones de frecuencias estadísticas, dentro de los cuadros estadísticos. Para ello, se utilizaron estadísticas descriptivas a través del cálculo de las frecuencias absolutas, proporciones y porcentajes, apoyados en la interpretación de medidas de tendencia central, además se establecieron los parámetros mínimos y máximos de las categorías a través de las puntuaciones de las alternativas de respuesta de los instrumentos arrojaron.

Para procesar los datos y analizarlos, se determinaron cinco categorías para el análisis, establecidas a partir del baremo de interpretación, este se construyó sobre la base de las puntuaciones utilizadas en la escala, la cual va de 1 a 5, ubicándose en los rangos Nunca (1- 1.79), Rara vez, (1.80 - 2.59), Ocasionalmente (2.60 3.39), Frecuentemente (3.40-4.19) y Muy Frecuente $(4.20-5)$.

\section{RESULTADOS}

Este punto, está referido a los resultados obtenidos en el cuestionario sobre las técnicas de actualización organizacional, y la discusión que se desprende del análisis de los mismos resultados. Los datos se muestran en una tabla de doble entrada; en cada indicador se calcularon las frecuencias absolutas y porcentuales por cada ítem, para luego construir la tabla en función de cada indicador. Además, se calcularon los promedios, los cuales se relacionaron con el baremo de interpretación que se muestran a continuación en la tabla 1, para luego evaluar sus principales tendencias.

Tabla 1. Técnicas del desarrollo organizacional

\begin{tabular}{|c|c|c|c|c|c|c|c|c|c|c|c|c|c|c|}
\hline \multirow{3}{*}{ Indicador } & \multirow{3}{*}{ Ítems } & \multicolumn{10}{|c|}{ Alternativas De Respuestas } & \multicolumn{3}{|c|}{ Promedios } \\
\hline & & \multicolumn{2}{|c|}{ Siempre } & \multicolumn{2}{|c|}{$\begin{array}{c}\text { Casi } \\
\text { Siempre }\end{array}$} & \multicolumn{2}{|c|}{ A Veces } & \multicolumn{2}{|c|}{$\begin{array}{l}\text { Casi } \\
\text { Nunca }\end{array}$} & \multicolumn{2}{|c|}{ Nunca } & \multirow[t]{2}{*}{ Ítems } & \multirow[t]{2}{*}{ Indic. } & \multirow[t]{2}{*}{ Dimen. } \\
\hline & & $\mathrm{FA}$ & $\%$ & $\mathrm{FA}$ & $\%$ & FA & $\%$ & FA & $\%$ & $\mathrm{FA}$ & $\%$ & & & \\
\hline \multirow{3}{*}{$\begin{array}{c}\text { Aprendizaje } \\
\text { Organizacional }\end{array}$} & 1 & 33 & 83 & 2 & 14 & 3 & 8 & 2 & 5 & 0 & 0 & 5 & \multirow{3}{*}{4,7} & \multirow{12}{*}{4,03} \\
\hline & 2 & 34 & 85 & 1 & 3 & 3 & 8 & 2 & 5 & 0 & 0 & 5 & & \\
\hline & 3 & 32 & 80 & 3 & 8 & 4 & 10 & 1 & 3 & 0 & 0 & 5 & & \\
\hline \multirow{3}{*}{$\begin{array}{c}\text { Gestión del } \\
\text { Conocimiento }\end{array}$} & 4 & 31 & 78 & 3 & 8 & 3 & 8 & 2 & 5 & 1 & 3 & 5 & \multirow{3}{*}{4,5} & \\
\hline & 5 & 35 & 88 & 3 & 8 & 1 & 3 & 1 & 3 & 0 & 0 & 5 & & \\
\hline & 6 & 25 & 63 & 6 & 15 & 3 & 8 & 3 & 8 & 3 & 8 & 4 & & \\
\hline \multirow{3}{*}{$\begin{array}{l}\text { Construcción } \\
\text { de Equipos }\end{array}$} & 7 & 8 & 20 & 4 & 10 & 4 & 10 & 3 & 8 & 21 & 53 & 2 & \multirow{3}{*}{3,8} & \\
\hline & 8 & 33 & 83 & 3 & 8 & 1 & 3 & 2 & 5 & 1 & 3 & 5 & & \\
\hline & 9 & 31 & 78 & 3 & 8 & 2 & 5 & 4 & 10 & 0 & 0 & 5 & & \\
\hline \multirow{3}{*}{ Socialización } & 10 & 12 & 30 & 8 & 20 & 12 & 30 & 8 & 20 & 0 & 0 & 3 & \multirow{3}{*}{3,5} & \\
\hline & 11 & 24 & 60 & 6 & 15 & 9 & 23 & 1 & 2 & 0 & 0 & 3 & & \\
\hline & 12 & 12 & 30 & 8 & 20 & 9 & 22 & 11 & 28 & 0 & 0 & 3 & & \\
\hline
\end{tabular}

En cuanto a las técnicas de desarrollo organizacional en las instituciones de educación superior, su dimensión estima un promedio de 4,03, considerando que frecuentemente se aplican técnicas de desarrollo organizacional. Para ello se involucraron cuatro indicadores; el primero denominado aprendizaje organizacional, su tendencia se orientó a la aplicación de esta técnica en las universidades, dado que sus resultados fueron $83 \%, 85 \%$ y $80 \%$ para la alternativa siempre. Su promedio fue de 4,7 , estimando que esta se aplica muy frecuentemente. Sus ítems incluyeron: el primero (ítem 1) que cuestionaba a los docentes sobre si la institución está orientada a la búsqueda de una mayor eficiencia organizacional, el segundo (ítem 2) pregunta sobre si el aprendizaje organizacional dentro de la institución proporciona oportunidades para alcanzar soluciones efectivas; y el tercero (ítem 3), detalla si está la capacidad de la institución para adaptarse a los cambios. 
El indicador gestión del conocimiento, estuvo asociado a tres ítems, tales como: si recibe respuesta de la institución a sus necesidades docentes en cuanto al desarrollo del conocimiento (ítem 4); si la institución pone a disposición del docente en forma clara y eficaz, los conocimientos explicativos de los procesos (ítem 5); además, si al gestionar la actualización tecnológica, a través del conocimiento se promueve un mejor funcionamiento de esta. A ello, $78 \%, 88 \%$ y $63 \%$ de la población respondió a la alternativa siempre a los tres ítems respectivamente. Aunado a ello el promedio final de este indicador fue de 4,5.

Asimismo, para el indicador construcción de equipos sus respuestas para el ítem 7, asociado a si la institución promueve actividades grupales para incrementar la confianza dentro del equipo de trabajo, fueron de $53 \%$ en la opción nunca, no obstante, para los otros ítems la alternativa siempre fue la más elegida con $83 \%$ para el ítem 8 sobre si utilizan las actividades de grupo para incrementar la confianza entre los miembros del equipo, suelen incluir la fijación de metas y $78 \%$ para el ítem 9, el cual cuestiona si las actividades para formación docente en actualización tecnológica, se realizan en función de reforzar los niveles de conocimiento grupal en el ámbito tecnológico. Sus tendencias apuntan a que en las instituciones de educación superior objeto de estudio se promueven los equipos de trabajo y que estos se utilizan para promover el conocimiento colectivo dentro de los grupos en cuanto a la adecuación tecnológica en el espacio educativo, puesto que su promedio es de 3,8, determinándolo con relación al baremo de interpretación de la variable.

El último indicador es la socialización, cuyas tendencias apuntan hacia la existencia de un proceso de socialización, pero este debe ser generado por la propia institución dado que casi un tercio $(30 \%)$ de la población seleccionó la opción siempre para el ítem 10, y 20\% (acumulando a la mitad de la muestra) lo consideró casi siempre, en virtud de que las instituciones objeto de estudio, les provee inducción para conocer los valores, normas y conductas requeridas para participar en la organización como miembro de esta. Además, para el ítem 11, 60\% asumió la opción siempre, y 15\% manifestó casi siempre sobre si, al no estar familiarizados con la cultura organizacional, se podría perturbar las creencias y costumbres que se han desarrollado. Por otra parte, el ítem 12, genera las tendencias respecto a si se promueve la adaptación de sus miembros a la cultura de la institución. A ello $30 \%$ contestó la opción siempre, y $20 \%$ casi siempre. Su promedio final es de 3,5.

De acuerdo a los resultado expresados anteriormente, se perfila la caracterización de las técnicas de desarrollo organizacional en instituciones de educación superior, destacando la prevalencia del aprendizaje organizacional, como elemento clave para promover las acciones del conocimiento en el docente, considerando que hay una aplicación continua y coherente de actualización tecnológica, capacitación continua, procesos de integración a las plataformas virtuales, manejos de los OVA, dichos procesos se han establecido para impulsar una mayor eficiencia organizacional, dicho esto, los investigadores han considerado que el aprendizaje organizacional como técnica del DO, proporciona oportunidades para alcanzar soluciones efectivas, en virtud de generar la capacidad de las instituciones para adaptarse a los cambios.

En este orden de ideas, Robbins y Judge (2013), consideran que una organización de aprendizaje es aquella que ha adquirido la capacidad continua de adaptarse y cambiar; tal como aprenden los individuos, también lo hacen las organizaciones. A su vez, García et al (2018), manifestaron que todas las organizaciones aprenden, aunque esa tendencia hacia el aprendizaje sea consciente o inconscientes, es decir que las organizaciones de aprendizaje tienen tendencia al doble ciclo, esto se refiere a que cuando se encuentra un error, se corrige de manera tal que implica la modificación de los objetivos, políticas y rutinas estándar de la organización.

Siguiendo con la caracterización de las técnicas, se apunta hacia la gestión del conocimiento, destacado al respecto, que las instituciones de educación superior (Universidad del Atlántico y Universidad de Sucre), tienen como objetivo estratégico dar respuesta a las necesidades del docente, por lo cual se avocan a promover el desarrollo del conocimiento, su actualización, así como que el docente pueda contar con una habilidad integral en gestión tecnológica; para ello ponen a disposición del docente en forma ordenada, y eficaz, los conocimientos explicativos de los procesos, promoviendo el mejor funcionamiento de esta.

Esta información se respalda con los preceptos de Nonaka y Takeuchi (2007), donde plantea que la gestión del conocimiento, es una disciplina emergente que tiene como objetivo generar, compartir y utilizar el conocimiento tácito (Know-how) y explícito (formal) existente en un determinado espacio, para dar respuestas a las necesidades de los individuos y de las comunidades en su desarrollo, centrado en la necesidad de administrar el conocimiento organizacional, al mismo tiempo el aprendizaje organizacional como mecanismos claves para el fortalecimiento de una región o espacio en relación con las visiones de futuro que van a determinar sus planes estratégicos de desarrollo en el mediano y largo plazo. Seguidamente se analiza el indicador construcción de equipos, en este ámbito en las instituciones de educación promueven los equipos de trabajo, utilizando actividades de grupo para incrementar la confianza entre los miembros del equipo, las cuales suelen incluir la fijación de metas; pero además se hacen de acuerdo con el propósito del esfuerzo del desarrollo. 
Seguidamente se analiza el indicador construcción de equipos, enfatizando que en las instituciones de educación se promueven los equipos de trabajo, a través de la ejecución de actividades de grupo para incrementar la confianza entre los miembros del equipo, en este contexto se incluyen fijación de metas, que respondan a las necesidades de los docentes, dar respuesta a las situaciones que desde el contexto externo e interno surgen como acciones del pensamiento creativo y divergente de la acción del docente, pero además se hacen de acuerdo con el propósito del esfuerzo del plan estratégico institucional (PEI).

Atendiendo a estas consideraciones, Robbins y Judge (2013), expresan que la construcción de equipos se puede aplicar en el interior de grupos o a nivel de relaciones entre grupos cuando las actividades son interdependientes. Como resultado, el interés se relaciona con las aplicaciones a las familias organizacionales (grupos de mando), así como a comités, equipos de proyectos, equipos autoadministrados y grupos de tareas. Igualmente, para Hersey, Blanchard y Johnson (2012), los integrantes de un equipo diagnostican la forma en la cual trabajan juntos y planean cambios para mejorar su eficacia, por ende, una organización comprende muchos equipos de trabajo diferentes, gran parte de su éxito depende de la eficacia con que estos grupos y sus integrantes pueden trabajar juntos, tal y como se evidencia en las instituciones objeto de estudio, existen diferentes grupos y entre ellos subgrupos, que se orientan a desarrollar acciones que les facilite cumplir con su rol de educar.

Dentro de estas perspectivas, en las universidades públicas de la región caribe, se está fomentando la construcción de equipos de trabajo como base para el desarrollo organizacional, empalmando en estas tendencias los procesos de conocimiento tecnológico, dando respuesta a las expectaciones de los estudiantes, lo que a su vez facilita la formación en actividades digitales, trabajaos en línea, manejo de OVA, salas virtuales, entre otras. Todo lo expresado encauzado al rendimiento institucional, trayendo como consecuencia un mayor desempeño del docente.

Finalmente, la otra técnica a caracterizar es la socialización, concepto que desde algunas perspectivas podría quedar desfasada de esta diatriba de procesos, no obstante, se hace relevante como el proceso de socialización del docente puede trascender hacia la actualización tecnológica y al manejo de los procesos virtuales. En este sentido, se evidencia que las instituciones de educación superior objeto de estudio, tienen establecidos procesos de socialización, los cuales parten de acción de inducción semestral que les da a conocer al personal docente las políticas, normas y conductas requeridas para participar en la organización como miembro de esta, además de recalcar el conjunto de valores organizacionales. En este ámbito los docentes reciben información continua en forma virtual para refuerzo sobre las líneas que deben desarrollar, de acuerdo con esto se considera necesario familiarizarse con la cultura organizacional, para no perturbar las creencias y costumbres que se han desarrollado, promoviendo la adaptación de sus miembros a la cultura de esta, cumpliendo con las metas y objetivos plasmados en el PEI.

Atendiendo a estos preceptos Durán (2014), considera que la rapidez y eficacia de la socialización determinan la lealtad, el compromiso, la productividad de los empleados, así como su permanencia o salida. De tal manera que La estabilidad, sumada a la eficacia de las organizaciones dependerán de la habilidad que tengan éstas para socializar a sus componentes. Bajo este contexto, en las universidades públicas de la región caribe, se requiere promover un proceso de socialización a partir de la integración del docente con los proyectos educativos, esto permite garantizar la formación continua del docente y contextualizar las actividades hacia plataformas tecnológicas, lo cual será transportado a los estudiantes, así como a la comunidad en general, como parte de la responsabilidad social.

\section{CONCLUSIONES}

De acuerdo al trabajo presentado y a los resultados obtenidos, se pueden plantear las siguientes conclusiones principales:

En cuanto a las técnicas de desarrollo organizacional en las instituciones de Educación Superior, se demostró que una de ellas es el aprendizaje organizacional, dado que se organiza capacitación continua, se coordinan actividades con los docentes en proceso presenciales y virtuales, se busca integrar las actividades del docente con las actividades del estudiantes orinándose a la búsqueda de una mayor eficiencia organizacional, en esa línea se destaca que esta técnica proporciona oportunidades para alcanzar soluciones efectivas, lo que resalta que las instituciones de educación superior puedan adaptarse a los cambios.

Con respecto a la gestión del conocimiento, en las instituciones se gestiona a través de planes que están organizados desde el proceso de planeación y talento humano, pero accionada hacia los objetivos del PEI, donde el estudiante sea el beneficiario. En este sentido, se pudo constatar que se busca dar respuesta a las inquietudes de los individuos en cuanto al conocimiento en los procesos y actividades que se relaciones con plataformas tecnológicas y virtuales, destacando que son opciones que se generan en la educación del siglo 
XXI, como línea estratégica de desarrollo. De esta manera se pone a disposición del docente en forma ordenada, y eficaz un modelo de ejecución de tareas y actividades, que les permita coordinar y ejecutar las salas virtuales, los OVA, las conferencias, entre otro. Además, vincular los conocimientos explicativos de los procesos, de tal manera de obtener el mejor funcionamiento de las instituciones en estudio.

Por otra parte, se promueven los equipos de trabajo, gestionando actividades de grupo para incrementar la confianza entre los miembros del equipo, se incluye la fijación de metas y posterior la evaluación del cumplimiento de dichas metas; pero además se hacen de acuerdo con el propósito del esfuerzo del desarrollo de los procesos y las acciones establecidas en el PEI y los programas académicos, de tal manera que la construcción de equipos se aplica en las facultades, en los programas académicos y entre ellos, generando cohesión y colaboración.

Consecuentemente, la socialización es una técnica que respalda un proceso de desarrollo organizacional, dado que los docentes deben alinearse a una línea institucional, donde prevalezcan los valores, políticas y normas, además de actividades de integración curricular. En este orden de ideas, la tendencia incluye que en las instituciones objeto de estudio cuentan con programas de inducción y seguimiento, que permiten viabilizar los indicadores de gestión del docente. Indudablemente que una de las bases fundamentales de una organización es que el docente debe conocer los procesos requeridos para participar en la organización como miembro de esta, por lo cual se hace necesario familiarizarse con la cultura organizacional.

Bajo este contexto, en las universidades públicas de la región caribe, se promueve un proceso de socialización a partir de la integración del docente con los proyectos educativos, esto permite garantizar la formación continua del docente, lo cual será transportado a los estudiantes, así como a la comunidad en general.

\section{REFERENCIAS}

Alles, M., y Rodríguez, A., Intellectual structure of human resources management research: A bibliometric analysis of the journal Human Resource Management, 1985-2005. doi: https://doi.org/10.1002/asi.20947, Journal of the American Society for Information Science and Technology 60(1), 161-175 (2011)

Bates, A. y Sangrá, A., Managing technology in higher education: strategies for transforming teaching and learning, John Wiley \& Sons, (2011)

Cummings, T. y Worley, C., Organization development and change. Cengage learning (2014)

Durán, S., Promoción de las instituciones educativas bajo el contexto de la interculturalidad, Revista Arbitrada Formación Gerencial, ISSN: 1690-074X, 13(2), (2014)

García J., Durán, S., Parra, M. y Martínez, H., Inserción, integración y equidad en el ámbito laboral: escenario empresarial posconflicto en Colombia, Revista de ciencias Sociales, ISSN: 1315-9518, 24(3) 36-49 (2018)

Guizar, R., Desarrollo organizacional principios y aplicaciones. Séptima edición, Editorial McGraw Hill. México DF, México (2013)

Hernández, I., Parra, M. y otros 4 autores, Comportamiento Organizacional Ciudadano (COC) como ejemplo de participación generador de un diálogo de saberes, https://www.revistaespacios.com/a18v39n07/a18v39n07p22.pdf, ISSN: 0798-1015, Revista Espacios, 39(7), 22-36 (2018)

Hernández, L., Jiménez, G. y otros 3 autores, Characterization of the use of the internet of things in the institutions of higher education of the city of region caribe and its metropolitan area. In: Stephanidis C. (eds) HCl International 2018 Posters' Extended Abstracts. $\mathrm{HCl}$ 2018. Communications in Computer and Information Science, vol 852. Springer, Cham (2018)

Hernández, L., Balmaceda, N. y otros 5 autores, Optimization of a wifi wireless network that maximizes the level of satisfaction of users and allows the use of new technological trends in higher education institutions. In: Streitz N., Konomi S. (eds) Distributed, Ambient and Pervasive Interactions. doi: https://doi.org/10.1007/978-3-030-21935-2_12, HCII 2019. Lecture Notes in Computer Science, Springer, Cham, vol. 11587 (2019)

Hersey, P., Blanchard, K. y Johnson, D., Management of organizational behavior (Vol. 9). Upper Saddle River, NJ: Prentice hall (2012)

Kaware, S. y Sain, S., ICT application in education: an overview. International Journal of Multidisciplinary Approach \& Studies, ISSN: 2348 - 537X, 2(1), 25-32 (2015)

Martínez, O., Steffens, E., Ojeda, D., y Hernández, H., Estrategias pedagógicas aplicadas a la educación con mediación virtual para la generación del conocimiento global, doi: https://dx.doi.org/10.4067/S0718-50062018000500011, Formación Universitaria, 11(5), 11-18 (2018)

Matukhin, D. y Zhitkova, E., Implementing blended learning technology in higher professional education. Procedia-Social and Behavioral Sciences, 206, 183-188 (2015)

Moreno, M., Tezón, M. y otros 3 autores, Autoestima: desarrollo de la autonomía personal en estudiantes del área técnica, https://www.revistaespacios.com/a18v39n46/a18v39n46p06.pdf, ISSN: 0798-1015, Revista Espacios, 39(46), 6-11 (2018) 
Moreno, Z., Parra, M., y otras 3 autoras., Importancia del pensamiento estratégico y acciones estratégicas para impulsar el emprendimiento social en las universidades venezolanas,

https://www.revistaespacios.com/a17v38n45/a17v38n45p04.pdf, ISSN: 0798-1015, Revista Espacios, 38(1) (2017)

Nonaka, I. y Takeuchi, H., The knowledge-creating company, Harvard business review, 85(7/8), 162 (2007)

Parra, M, Visbal, O., Durán, S. y Badde, G., Calidad de la comunicación y actitud de los empleados ante procesos de cambio organizacional Interdisciplinaria, doi: https://doi.org/10.16888/interd.2019.36.1.11, Centro Interamericano de Investigaciones Psicológicas y Ciencias Afines, 36(1), 155-170 (2019)

Rivera, J., Lay, N. y otros 6 autores, Programa de entrenamiento para desarrollar habilidades sociales en estudiantes universitarios, https://www.revistaespacios.com/a19v40n31/a19v40n31p10.pdf, ISSN: 0798-1015, Revista espacios, 40 (31), 10-25 (2019)

Robbins, S. y Judge, T., Organizational behavior, Pearson education limited (2013)

Sánchez, M., García, J., Steffens, E., y Hernández, H., Estrategias pedagógicas en procesos de enseñanza y aprendizaje en la educación superior incluyendo tecnologías de la información y las comunicaciones, doi: https://dx.doi.org/10.4067/S0718-07642019000300277, Información Tecnológica, 30(3), 277-286 (2019).

Villarreal, S., García, J., Hernández, H. y Steffens, E., Competencias docentes y transformaciones en la educación en la era digital, doi: https://dx.doi.org/10.4067/S0718-50062019000600003. Formación Universitaria, 12(6), 3-14, (2019)

Viloria, A., López, J., y otros 5 autores, Determinating student interactions in a virtual learning environment using data mining, doi: https://doi.org/10.1016/j.procs.2019.08.082, Procedia Computer Science, 155, 587-592 (2019)

Viloria, A., Martínez, D. y otros 6 autores, Optimization of flow shop scheduling through a hybrid genetic algorithm for manufacturing companies. In: Pandian A., Ntalianis K., Palanisamy R. (eds) Intelligent Computing, Information and Control Systems. ICICCS 2019. Advances in Intelligent Systems and Computing, doi: https://doi.org/10.1007/978-3-030-304652_3, Springer, Cham, Vol 1039 (2020) 
